International Journal of Engineering \& Technology, $7(1.1)(2018) 699-703$
International Journal of Engineering \& Technology
Website: $w$ ww.sciencepubco.com/index.php/IJET
Research paper

\title{
A novel method for minimizing distortions in endoscopy medical images
}

\author{
R. Sathiya ${ }^{1 *}$, Dr.R. Kalaimagal ${ }^{2}$ \\ ${ }^{1}$ Scholar in Computer Science, Research and Development Centre, Bharathiyar \\ University, Coimbatore, India. \\ ${ }^{2}$ Assistant Professor in Department of Computer Science, Government Arts College, \\ Nandhanam, Chennai, India \\ *Corresponding author E-mail: sathiyaat@gmail.com
}

\begin{abstract}
A short prologue to the historical backdrop of endoscopy we outline the distinctive strategies which exist to perform endoscopic techniques. Medicinal endoscope creature has the attributes as very exactness, utilitarian adaptability and cost effectiveness, which makes it the aide of therapeutic, analyze and additionally the establishment of long-remove transmission of restorative pictures .In this paper, real time endoscopic optical rationality tomography (EOCT) imaging framework. A few mechanical advancements are presented that enhance EOCT proficiency and execution. By utilizing EOCT framework, clinical investigations in first stage the throat, intestine, duodenum, ileum, colon, and rectum of patients with typical endoscopic discoveries were inspected. Tiny structures, for example, organs, veins, pits, awful, and graves were likewise watched. The mutilation of medicinal picture, for example, fluffy key points of interest of tissues might show up affected by the intertwining filtering innovation, which may cause misdiagnoses. De-interweaving system to kill mutilation is basic. At last, as indicated by the significant reason of picture intertwining twisting in medicinal endoscope framework, versatile de-joining calculation is utilized to wipe out the mutilation with.
\end{abstract}

Keywords: EOCT pictures, Optimization, Interpolation bending expulsion, contortion remedy.

\section{Introduction}

Through fast improvement of endoscopic Surgery Techniques and Minimally Invasive Surgical Techniques, Medical Electronic Endoscope has presently turned out to be broadly appropriate therapeutic instruments. It is framed by conventional endoscopic procedures, present day PC innovation and miniaturized scale hardware strategies. The three primary portions of Medical Electronic Endoscope is endoscopy, video data framework focus then TV screen. In endoscopy Doctors can straightforwardly watch the morpha and pathology of interior organs and give analyze. What's more, the traded or spared pictures can be prepared for assist determination and treat. The restorative group has just achieved an agreement of the preferred standpoint. These days restorative endoscopes are uncontrollably utilized as a part of gastro scope, right now degree and colonoscopy, and so on. Specialists get the genuine picture of patient tissues in the test to provide more exact conclusions which can diminish the patients' agony affected by incorrectly identify. Furthermore, beforehand as soon as experienced person specialist playing out the task of a troublesome in addition confused ailment, there are dependably be numerous understudies the person want to study or participate in it.

In any case, the utmost operation theatre can't address their issues. By and by this issue can be unraveled by report all procedure of the task by camcorder. Assistants can have video match up study or concentrate later by arrange procedures and picture preparing strategies. Be that as it may, the development of human's organ will cause transient obscured of the picture got by entwined examine endoscope. Particularly at the minimal parts, there would be a genuine draw seat marvel, which caused the video terminal showed not unmistakably at those parts.

This gives higher troubles of medicinal endoscopy frameworks' post-acknowledgment handling, which requires high clearness and precision. It brings about incredibly lessened acknowledgment impact and influences the demonstrative precision.

In this manner we have to embrace de-interweaving innovation to dispense with picture entwining bending. Inside the previous two decades there are different de-entwining techniques rose, for example, basic spatial-area introduction, directionality separating and propelled dynamic pay addition. Also, some of these strategies have just been uncontrollably utilized as a part of video items. This paper will right off the bat investigate the reason of interweave examining picture joining bending in light of the rule of intertwined check.

Picture preparing is handling of pictures utilizing scientific tasks by utilizing any assortment of flag preparing for which the info is a picture, a progression of pictures, or a video, for example, a picture taker video outline; the yield of picture preparing is either a picture or an arrangement of attributes or parameters identified with the picture. Most picture handling methods include regarding the picture as a dimensional flag and applying standard flag preparing strategies to it. Pictures are additionally handled as threedimensional signs where the third-measurement being time or the z-pivot. 
Picture preparing is brought out through advanced picture handling, yet optical and simple picture handling likewise are conceivable. This article is about general systems that apply to every one of them. The ownership of pictures (creating the info picture in any case) is alluded to as imaging.

Straightforwardly associated with picture handling are PC designs and PC vision. In PC designs, pictures are physically produced using physical models of items, conditions, and lighting, instead of being gained (by means of picture catching gadgets, for example, cameras) from common scenes, as in for the most part energized motion pictures. At that point, Computer vision is regularly viewed as abnormal state picture preparing in relationship with a machine/PC/programming means to decode the physical substance of a picture or a grouping of pictures (e.g., recordings or 3D fullbody attractive reverberation checks).

In present day sciences and advancements, pictures additionally extend significantly more extensive degrees because of the consistently developing significance of logical representation (of frequently expansive scale complex logical/test information). Cases contain microarray information in hereditary research, or ongoing multi-resource portfolio exchanging fund.

Picture administration is an address to change over a account into computerized anatomy and plays out a few tasks, behindhand of accepting an upgraded account or to abstracted some admired abstracts from it. It is an affectionate of banderole adjustment as advice is crop a picture, agnate to video bend or photo and crop ability be account or qualities accompanying with that picture. For the most part in Image Processing framework pictures as two dimensional signs while applying effectively set flag preparing techniques to them.

Among picture preparing is quickly developing innovations today, with its applications in different parts of a business. Picture Processing frames center research territory inside designing and software engineering disciplines as well. Picture preparing is fundamentally incorporates the three stages. They are

- Importing the picture with optical scanner or by computerized photography.

- $\quad$ Analyzing and controlling the picture which incorporate information pressure and picture enhancement and spotting designs that are not to human eyes like satellite photos.

- $\quad$ Output result can be twisted picture or report that depends on picture examination.

The motivation behind picture handling is isolated into 5 gatherings. They are:

1. Representation - Examine the items that are not unmistakable.

2. Picture honing and reclamation - To make a superior picture.

3. Picture recovery - Try to discover the picture of intrigue.

4. Estimation of example - Measures different protests in a picture.

5. Picture Recognition - Differentiate the articles in a picture.

The two kinds of strategies are utilized for Image Processing they are Analog and Digital Image Processing. Simple or visual strategies of picture preparing can use for the printed copies like printouts and photos.

Advanced Processing systems help to control of the computerized pictures by methods for PCs. As awkward advice from imaging sensors from digital date contains insufficiency. To get over such birthmark and to get character of data, it needs to acquaintance altered periods of preparing. The three accepted stages that a advanced ambit of advice charge to acquaintance while utilizing computerized arrangement are Pre-preparing, advancement and show, abstracts extraction. Picture Processing Toolbox gives an entire arrangement of reference-standard calculations, capacities, and applications for picture preparing, investigation, representation, and calculation advancement. Picture Processing Toolbox bolsters a different arrangement of picture composes, including high powerful range, giga pixel determination, implanted ICC profile, and tomography. Perception capacities and applications let you investigate pictures and recordings, watch a locale of pixels, modify shading and difference, make forms or histograms, and control districts of premium (ROIs). The tool kit bolsters work processes for handling, showing, and exploring expansive pictures

\section{Image}

A picture alludes to a $2 \mathrm{D}$ light power work $\mathrm{f}(\mathrm{a}, \mathrm{b})$, where $(\mathrm{a}, \mathrm{b})$ speak to spatial directions then estimation of $f$ anytime $(a, b)$ is relative to the splendor or dim points of the picture by then.

- A computerized picture is a picture $f(a, b)$ that has been spoiled in cooperation with spatial directions and shine.

- The components of such an advanced exhibit are known as picture components otherwise pixels.

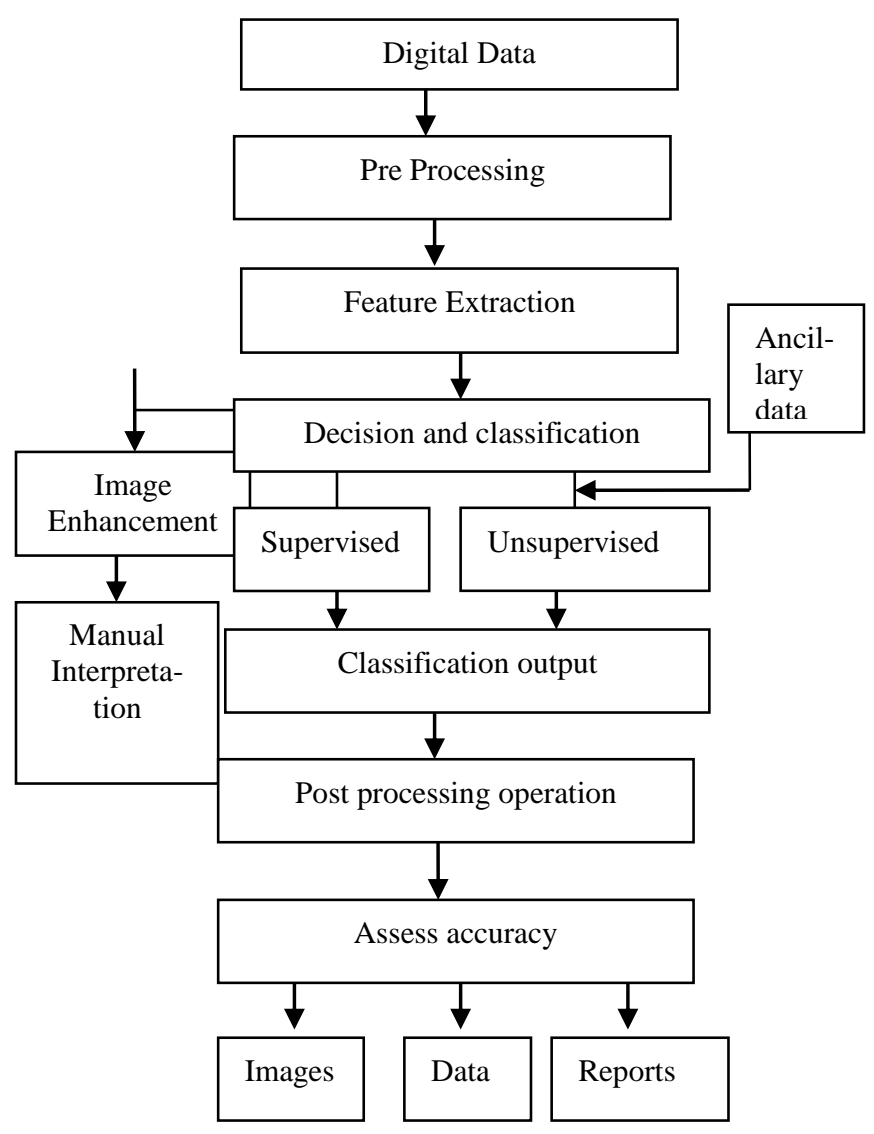

Fig1 Flow chart of image analyst 
- Computer processing is suitable for an photo $f(a, b)$ need to be digitalized in cooperation with spatially and amplitude.

- Photograph sampling is digitization of the spatial coordinates (a, b).

- Amplitude digitization is called grey-degree quantization.

- The garage and processing necessities boom quickly with the spatial resolution and the number of grey ranges.

- Example: a 256 grey-stage picture of size $256 \times 256$ occupies $64 \mathrm{k}$ bytes of reminiscence.

- Pictures of very low spatial decision generate a checkerboard effect.

- Using inadequate variety of grey stages in easy areas of a digital picture effects in false contouring.

\section{Overview}

A picture is an exhibit, or grid, of square pixels (picture components) orchestrated in sections and columns. In a (8-bit) dim scale picture each photo component has an allocated force that extents from0 to 255. A dim scale picture is the thing that individuals regularly calla highly contrasting picture, however the name underlines that such a picture will likewise incorporate numerous shades of dark.
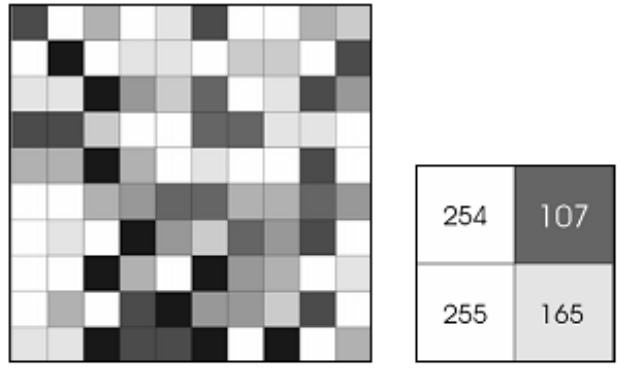

Fig 2 Gray scale image-Block wise representation

\subsection{Fundamental steps in image processing}

1.Picture securing: to acquire a computerized picture

2. Picture pre-preparing: to enhance the picture in conduct that builds the odds for accomplishment of alternate procedures.

3. Picture division: to parcels an info picture into its essential parts or articles.

4. Picture portrayal: to change over the info information to a shape appropriate for PC preparing.

5. Picture depiction: to extricate highlights that outcome in some quantitative data of interest or highlights that are fundamental for separating one class of articles from another.

6. Picture acknowledgment: to allot a mark to a question in light of the data gave by its descriptors.

7. Picture elucidation: to apportion importance to a group of perceived items.

\subsection{Hues}

In science communication, the main two color spaces are RGB and CMYK.

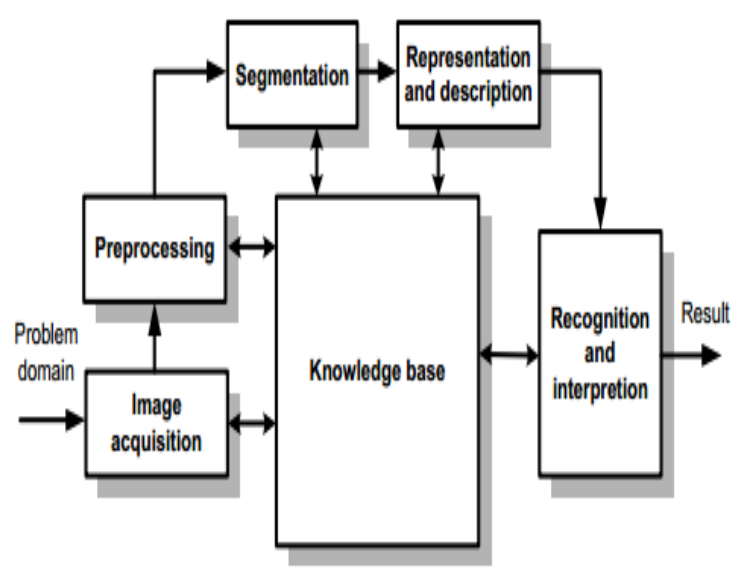

Fig 3 Block diagram using knowledge based technique

\subsection{RGB}

The RGB shading model is nearly to the way we see shading with the $r, g$ and $b$ receptors in our retinas. RGB utilizes stabilizer shading blending and is the essential shading model utilized as a part of TV or some other medium that undertaking shading with light. The essential shading model is utilized as a part of PCs and for web illustrations, however it can't be utilized for print creation. The auxiliary shades of RGB - cyan, maroon, and yellow- are created also two of the essential hues (red, green or blue) and without the third shading. Red and green consolidate to make yellow, green and blue to make cyan, and blue and red frame Magenta

\section{Research methodology}

\subsection{Enhanced distortion correction algorithm}

The part of the non-linearity redress calculation is to amend the uncertain directions of occasions (pixels) in the yield picture. These loose facilitates make the info information that will be bolstered to the adjustment calculation, while the yield of the calculation will speak to the redressed organizes. The revision calculation conveys a rectification cluster, in this exhibit the lists in $\mathrm{x}, \mathrm{y}$ headings are the uncorrected directions, and the clusters component will be the remedied areas.

The multi-resound reference examine, be that as it may, encodes the stage development from B0 in homogeneity, as well as from concoction move and readout slope vortex streams. There is the extra preferred standpoint that no stage unwrapping is basic yet regions of high $\mathrm{B} 0$ in homogeneity. From the Fourier move hypothesis, the dislodging (in pixels) of every pixel in the stage encode course is given by the quantity of insurgencies of de-staging over the procurement time.

\subsection{Interpolation distortion removal}

In a left-gave facilitate framework with the starting point at the upper left corner of a picture, the directions of a point in the ith line and $\mathrm{j}$-th section of the picture are $(\mathrm{j}, \mathrm{I})$. In the event that the unit of time is characterized as one picture push, the quantity of columns in a picture is $\mathrm{N}$, and the between pixel delay is $\mathrm{d}$, at that point the imaging moment of a point in the I-th line of the k-th picture is $(\mathrm{k}-1)(\mathrm{N}+\mathrm{d})+\mathrm{i}$.

To evacuate moving screen contortions in a picture, an amendment strategy ought to produce another casing in which all pixels are imaged in the meantime moment. The time moment when the center line of the first casing is imaged is frequently picked as the reference time. The most extreme revision of a point would thus 
be able to be limited. For the k-th outline, every pixel in the revised casing ought to be imaged at time moment $(\mathrm{k}-1)(\mathrm{N}+\mathrm{d})+\mathrm{N} / 2$. A point caught at time moment $(\mathrm{k}-1)(\mathrm{N}+\mathrm{d})+\mathrm{i}$ ought to be moved to another situation as though it is caught at time moment $(\mathrm{k}-$ 1) $(\mathrm{N}+\mathrm{d})+\mathrm{N} / 2$

\subsection{Optimization}

To enroll a source volume $\alpha$ to the objective volume T, we need to discover the estimations of the parameters of the pictures. To accelerate the twisting redress, we don't have any significant bearing the shine rectification amid the enhancement procedure. Here, we utilize a straightforward inclination rising enhancement system combined with a brilliant segment line streamlining strategy to boost Q

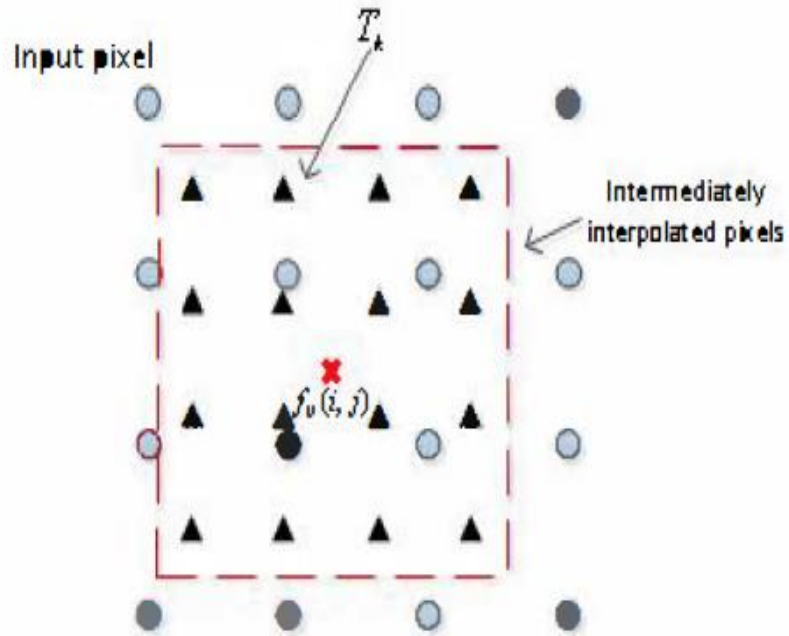

Fig 4: Interpolated pixels for the proposed enhanced distortion correction algorithm.

\subsection{Endoscopic OCT Methods}

The relative no trouble in executing rapid and a highdetermination OCT framework has provoked various endeavors to actualize OCT-based endoscopes. The essential outline of thought is to limit the measurement of the OCT test. To this end, the single-mode fiber is utilized for light transmission for the most part OCT frameworks is preferably suited for the reason. Other vital specialized qualities of OCT test are examining range, field of view, speed, and adaptability. OCT tests can be separated into two gatherings in light of their sweep modes are side-imaging and forward-imaging tests.

\subsection{Side-Imaging OCT}

Most of the endoscopic OCT frameworks are side-examining. In these frameworks, the OCT test light is transmitted from and gathered along the edge of the endo-scope. The general outline of test is comprises of a bar mirror or crystal appended to a turn get together to avoid the radiated light from the optical fiber tip out of a window in favor of the test. Side-imaging tests can be additionally isolated into two classes: 1) circumferential and 2) direct examining tests.

\subsection{Forward-Imaging OCT}

Rather than side-imaging OCT endoscopes, forward-imaging OCT endoscopes transmit and gather light before the test; consequently, it can be exceptionally helpful in giving the tissue basic data forward of a catheter test for picture direction amid surgery or gadget situation. Not at all like side-imaging OCT frameworks, the usage of a forward-imaging framework inside a limited test is all the more in fact testing; obviously, the forward-imaging tests reported so far are moderately huge in measurement.

\section{Results}

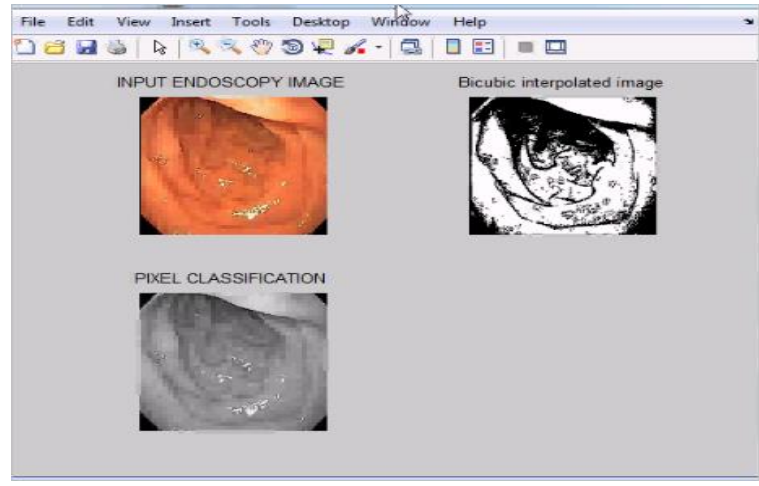

Fig 5. Classified image

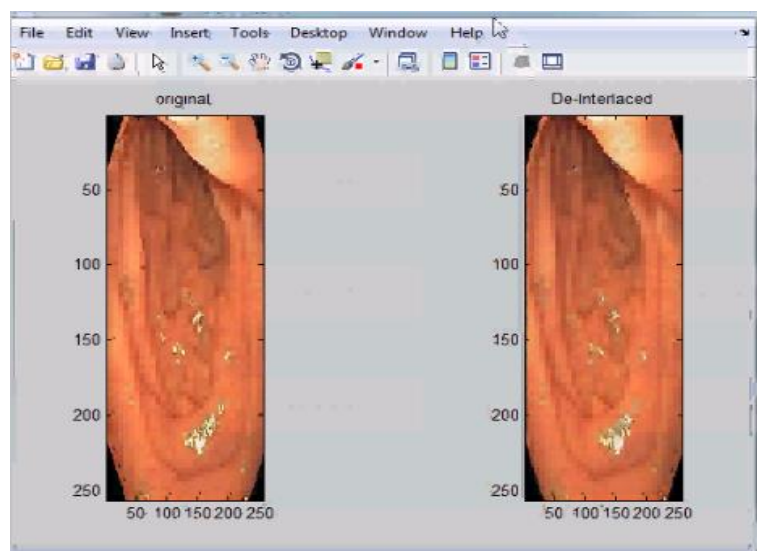

Fig 6. Original and destructured image

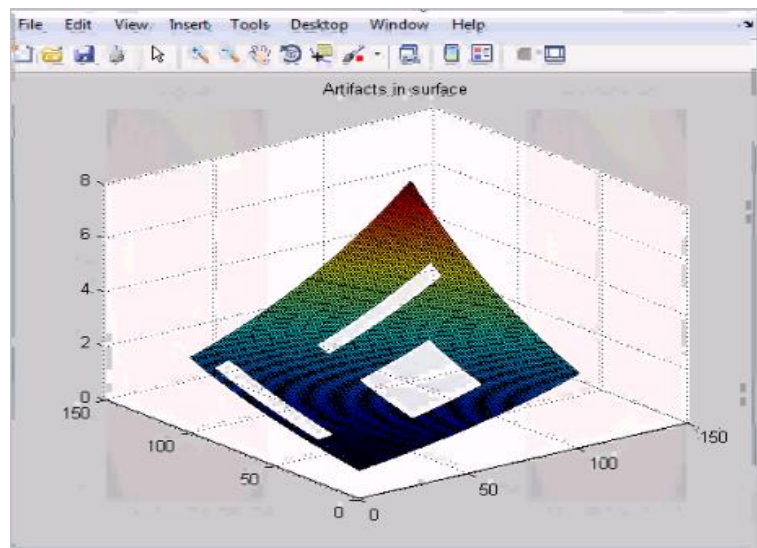

Fig 7. Artifacts in endoscopic

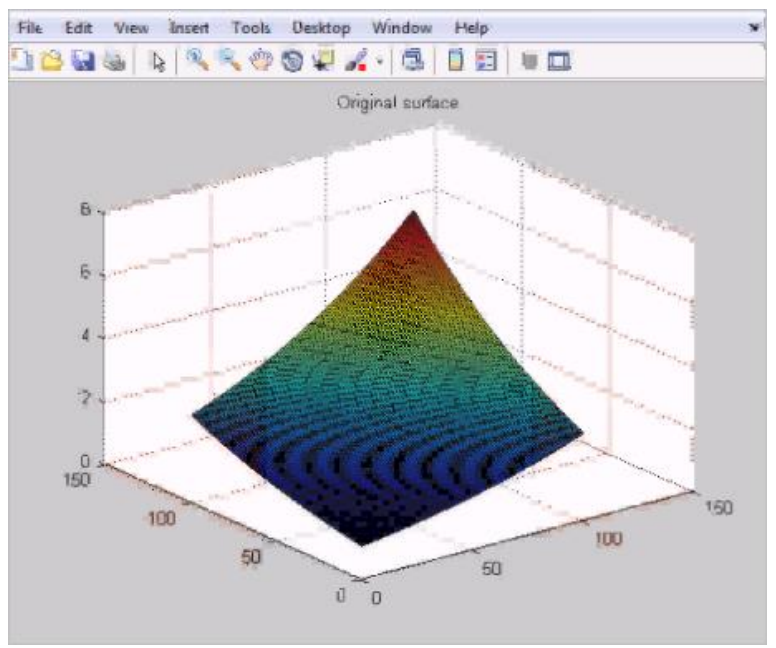

Fig 8. Artifact reduced surface 


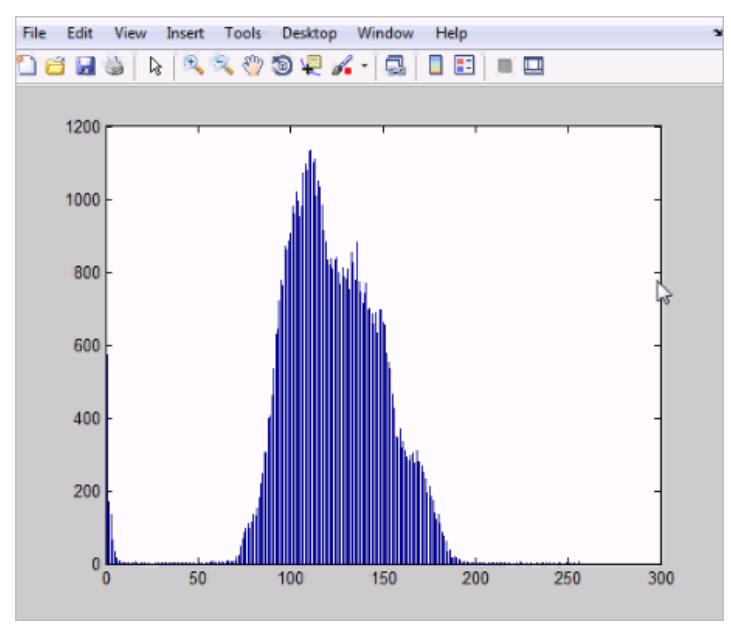

Fig. 9. Histogram equalization analysis

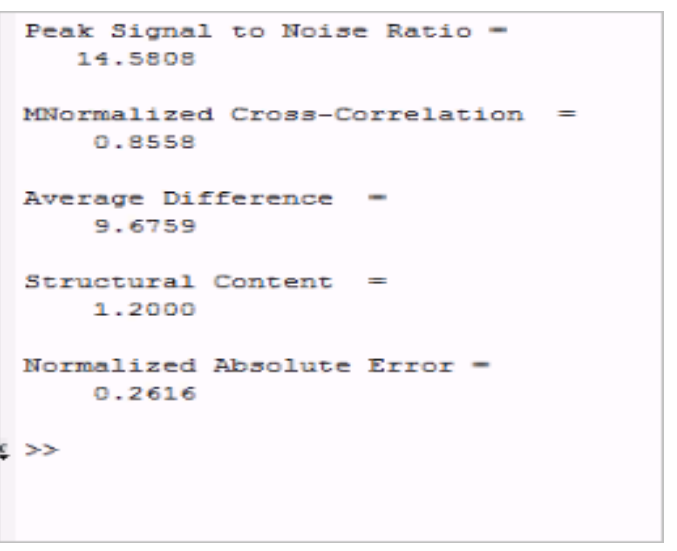

Fig. 10: Analysis of cross correlation and error

\section{Conclusion}

We provide details regarding the plan and starting clinical involvement with an ongoing endoscopic optical cognizance tomography (EOCT) imaging framework. A few mechanical advancements are presented that enhance EOCT effectiveness and execution. In introductory clinical examinations utilizing the EOCT framework, the throat, stomach, duodenum, ileum, colon, and rectum of patients with typical endoscopic discoveries were inspected. Infinitesimal structures, for example, organs, veins, pits, villi, and tombs were additionally watched. Optical cognizance tomography (OCT) is an imaging innovation that empowers micron scale, cross-sectional and three-dimensional (3D) imaging of test microstructure progressively. 3D imaging is acquired by playing out a two-dimensional output design at various transverse positions.

Endoscopic Three-dimensional OCT (3D-OCT) empowers intense techniques for picturing tissue engineering. 3D-OCT creates complete, volumetric informational indexes, which can be utilized to develop self-assertive cross-sectional pictures, projections along discretionary tomahawks, or 3D renderings like attractive reverberation imaging (MRI) or figured tomography (CT). The twisting of restorative picture, for example, fluffy key subtle elements of organs may show up caused by the intertwining filtering innovation, which may bring about misdiagnoses. De-joining strategy ought to be utilized as a part of medicinal endoscope framework, clearly entwining twisting occurs in the development zone of organs in restorative pictures caused by the standard of interweaved filter and the sporadic development of human's organs. Versatile de-interweaving strategy has straightforward estimation, and It keeps the clearness of static piece of the picture while just preparing the parts with development. The preparing reason for existing is attractive, so it's appropriate for down to earth application in restorative endoscope frameworks. De-intertwining system to dispense with mutilation is fundamental. At last, as per the real reason of picture entwining bending in restorative endoscope framework, versatile de-interweaving calculation is utilized to wipe out the twisting with. Hence we can get the unmistakable pictures by utilizing versatile de-joining calculation.

\section{References}

[1] Huang, D.; Swanson, E.A.; Lin, C.P.; Schuman, J.S.; Stinson, W.G.; Chang, W.; Hee, M.R.; Flotte, T.; Gregory, K.; Puliafito, C.A.; et al. Optical coherence tomography. Science 1991, 254, $1178-1181$.

[2] Fujimoto, J.G.; Pitris, C.; Boppart, S.A.; Brezinski, M.E. Optical coherence tomography: An emerging technology for biomedical imaging and optical biopsy. Neoplasia 2000, 2, 9-25.

[3] Fujimoto, J.G. Optical coherence tomography for ultrahigh resolution in vivoimaging. Nat. Biotechnol. 2003, 21, 1361-1367.

[4] Fujimoto, J.G.; Brezinski, M.E.; Tearney, G.J.; Boppart, S.A.; Bouma, B.; Hee, M.R.; Southern, J.F.; Swanson, E.A. Optical biopsy and imaging using optical coherence tomography. Nat. Med. 1995, 1, 970-972.

[5] Brezinski, M.E.; Tearney, G.J.; Bouma, B.E.; Izatt, J.A.; Hee, M.R.; Swanson, E.A.; Southern, J.F.; Fujimoto, J.G. Optical coherence tomography for optical biopsy. Properties and demonstration of vascular pathology. Circulation 1996, 93, 1206-1213.

[6] Tearney, G.J.; Brezinski, M.E.; Bouma, B.E.; Boppart, S.A.; Pitvis, C.; Southern, J.F.; Fujimoto, J.G. In vivo endoscopic optical biopsy with optical coherence tomography. Science 1997, 276, 2037-2039.

[7] Takada, K.; Yokohama, I.; Chida, K.; Noda, J. New measurement system for fault location in optical waveguide devices based on an interferometric technique. Appl. Opt. 1987, 26, 1603-1608.

[8] Gilgen, H.H.; Novak, R.P.; Salathe, R.P.; Hodel, W.; Beaud, P. Submillimeter optical reflectometry. IEEE J. Lightwave Technol. 1989, 7, 1225-1233.

[9] Youngquist, R.; Carr, S.; Davies, D. Optical coherence-domain reflectometry: A new optical evaluation technique. Opt. Lett. 1987, 12, 158-160.

[10] Swanson, E.A.; Huang, D.; Hee, M.R.; Fujimoto, J.G.; Lin, C.P.; Puliafito, C.A. High-speed optical coherence domain reflectometry. Opt. Lett. 1992, 17, 151-153.

[11] Swanson, E.A.; Izatt, J.A.; Hee, M.R.; Huang, D.; Lin, C.P.; Schuman, J.S.; Puliafito, C.A.; Fujimoto, J.G. In vivo retinal imaging by optical coherence tomography. Opt. Lett. 1993, 18, 1864-1866.

[12] Fercher, A.F.; Hitzenberger, C.K.; Kamp, G.; Elzaiat, S.Y. Measurement of intraocular distances by backscattering spectral interferometry. Opt. Commun. 1995, 117, 43-48.

[13] Choma, M.A.; Sarunic, M.V.; Yang, C.H.; Izatt, J.A. Sensitivity advantage of swept source and fourier domain optical coherence tomography. Opt. Exp. 2003, 11, 2183-2189.

[14] De Boer, J.F.; Cense, B.; Park, B.H.; Pierce, M.C.; Tearney, G.J.; Bouma, B.E. Improved signal-to-noise ratio in spectral-domain compared with time-domain optical coherence tomography. Opt. Lett. 2003, 28, 2067-2069.

[15] Leitgeb, R.; Hitzenberger, C.K.; Fercher, A.F. Performance of fourier domain vs. Time domain optical coherence tomography. Opt. Exp. 2003, 11, 889-894.

[16] Cense, B.; Nassif, N.; Chen, T.C.; Pierce, M.C.; Yun, S.; Park, B.H.; Bouma, B.; Tearney, G.; de Boer, J.F. Ultrahigh-resolution high-speed retinal imaging using spectral-domain optical coherence tomography. Opt. Exp. 2004, 12, 2435-2447. 